\title{
THE GREEN'S FUNCTIONS AND DENSITY FUNCTIONAL APPROACH TO VIBRATIONAL STRUCTURE IN THE PHOTOELECTRON SPECTRA OF MOLECULES
}

\begin{abstract}
We present the basis's of the new combined theoretical approach to vibrational structure in photoelectron spectra of molecules. The approach is based on the Green's function method and quasiparticle density functional theory (DFT). The density of states, which describe the vibrational structure in photoelectron spectra, is defined with the use of combined DFT-Green's-functions approach and is well approximated by using only the first order coupling constants in the one-particle approximation. Using the DFT theory leads to significant simplification of the calculation.
\end{abstract}

\section{INTRODUCTION}

A number of phenomena, provided by interaction of electrons with vibrations of the atomic nuclei in molecules or solids is manifested in the molecular photoelectron spectra. Indeed, the physics of the interaction of electrons with vibrations of the atomic nuclei in molecules or solids is more richer (c.f.[116]). One could mention here a great field of the resonant collisions of electrons with molecules, which are one of the most efficient pathways for the transfer of energy from electronic to nuclear motion. While the corresponding theory has been refined over the years with sophisticated and elaborate non-local treatments of the reaction dynamics, such studies have for the most part treated the nuclear dynamics in one dimension. This situation has resulted from the fact that, as the field of electron-molecule scattering developed, both experimentally and theoretically, the phenomena of vibrational excitation and dissociative attachment were first understood for diatomics, and it seemed natural to extend that understanding to polyatomic molecules using one-dimensional or single-mode models of the nuclear motion. However a series of experimental measurements of these phenomena in small polyatomic molecules have proven to be uninterpretable in terms of atomic motion with a single degree of freedom. Primary among these are potential-energy surfaces which describe the behavior of the electronic energy with respect to the locations of the nuclei, subject to the underlying Born-Oppenheimer or clamped nuclei approximation. From the ground- and excited-state wave functions one could in principle obtain all properties that arise from a solution to the vibrational Schrцdinger equation that gives the frequencies, and, with the derivatives of the dipole moment, the infrared intensities [1-6]. Electronic excited states are also accessible along with electronic and photoelectron spectra.

As it is often takes a place, the old multi-body quantum theoretical approaches, which have been primarily developed in a theory of superfluity and superconductivity, and generally speaking in a theory of solids, became by the powerful tools for develop- ing new conceptions in a theory of molecules [7-11]. Many of them offers a synthesis of cluster expansions, Brueckner's summation of ladder diagrams, the summation of ring diagrams Gell-Mann and an infinite-order generalization of many-body perturbation theory (MBPT). Using the quantum-field methods in molecular theory allowed to obtain a very powerful approach for correlation in many-electron systems.

The Green's method is very well known in a quantum theory of field, quantum theory of solids (c.f. $[7,8])$. Naturally, an attractive idea was to use it in the molecular theory. Returning to problem of description of the vibrational structure in photoelectron spectra of molecules, it is easily understand that this approach has great perspective (c.f. $[12,13])$. One could note that the experimental photoelectron (PE) spectra usually show a pronounced vibrational structure. Usually the electronic Green's function is defined for fixed position of the nuclei. As result, only vertical ionization potentials (V.I.P.'s) can be calculated [14]. The cited method, however, requires as input data the geometries, frequencies, and potential functions of the initial and final states. Since in most cases at least a part of these data are unavailable, the calculations have been carried out with the objective of determining the missing data by comparison with experiment. Naturally, the Franck-Condon factors are functions of the derivatives of the difference between the potential curves of the initial and final states with respect to the normal coordinates. To avoid the difficulty and to gain additional information about the ionization process, the Green's functions approach has been extended to include the vibrational effects in the photoelectron spectra. Nevertheless, there are well known great difficulties of the correct interpretation of the photoelectron spectra for any molecules.

Further let us remember that for larger molecules and solids, far more approximate but more easily applied methods such as density-functional theory (DFT) [15-17] or from the wave-function world the simplest correlated model MBPT are preferred [3]. Indeed, in the last decades DFT theory became by a great, quickly developing field of the modern quan- 
tum computational chemistry of molecules, solids. Naturally, this approach does not allow to reach a spectroscopic accuracy in description of the different molecular properties, nevertheless, the key idea is very attractive and can be used in new combined theoretical approaches.

Here we present the basis's of the new combined theoretical approach to vibrational structure in photoelectron spectra of molecules. The approach is based on the Green's function method (CederbaumDomske version) [12,13] and Fermi-liquid DFT formalism [11,14] (see also [18-23,25]). The density of states, which describe the vibrational structure in molecular photoelectron spectra, is calculated with the help of combined DFT-Green's-functions approach. In addition to exact solution of one-bode problem different approaches to calculate reorganization and many-body effects are presented. The density of states is well approximated by using only the first order coupling constants in the one-particle approximation. It is important that the calculational procedure is significantly simplified with using the quasiparticle DFT formalism. Thus quite simple method becomes a powerful tool in interpreting the vibrational structure of photoelectron spectra for different molecular systems.

2. The Hamiltonian of the system. The density of states in one-body and many-body solution

The quantity which contains the information about the ionization potentials and the molecular vibrational structure due to quick ionization is the density of occupied states [12]:

$$
N_{k}(\epsilon)=(1 / 2 \pi \hbar) \int d t e^{i \hbar^{-1} \epsilon t}\left\langle\psi_{0}\left|\mathrm{a}_{k}^{t}(0) a_{k}(t)\right| \psi_{0}\right\rangle,
$$

where $\left|\Psi_{0}\right\rangle$ is the exact ground state wavefunction of the reference molecule and $a_{k}(t)$ is an electron destruction operator, both in the Heisenberg picture. For particle attachment the quantity of interest is the density of unoccupied states:

$$
N_{k}(\epsilon)=(1 / 2 \pi \hbar) \int d t e^{i \hbar^{-1} \epsilon t}\left\langle\psi_{0}\left|\mathrm{a}_{\mathrm{k}}(\mathrm{t}) \mathrm{a}_{k}^{t}(0)\right| \psi_{0}\right\rangle
$$

Usually in order to calculate the value (1) states for photon absorption one should express the Hamiltonian of the molecule in the second quantization formalism (see [12,13]). The corresponding Hamiltonian is as follows:

$$
H=T_{\mathrm{E}}(\partial / \partial x)+T_{\mathrm{N}}(\partial / \partial X)+U(x, X),
$$

where $T_{\mathrm{E}}$ is the kinetic energy operator for the electrons, $T_{\mathrm{N}}$ is the kinetic energy operator for the nuclei, and $\boldsymbol{U}$ represents the interaction

$$
U(x, X)=U_{\mathrm{EE}}(x)+U_{\mathrm{NN}}(X)+U_{\mathrm{EN}}(x, X),
$$

where $\boldsymbol{x}$ denotes electron coordinates, $\boldsymbol{X}$ denotes nuclear coordinates, $U_{\mathrm{EE}}$ represents the Coulomb interaction between electrons, etc. Introducing the field operator:

$$
\Psi(R, \theta, x)=\sum \phi_{i}(x, R, \theta) a_{i}(R, \theta)
$$

where the $\boldsymbol{\phi}_{\text {, }}$ are Hartree-Fock (HF) one-particle functions and the $\boldsymbol{a}_{\boldsymbol{i}}$ are destruction operators for a $\mathrm{HF}$ particle in the " $\mathrm{i}$ " state, them the Hamiltonian in a occupation number representation is given as

$$
\begin{gathered}
H=H_{\mathrm{EN}}\left(R, \theta_{0}\right)+U_{\mathrm{NN}}\left(R, \theta_{0}\right)+T_{\mathrm{N}}(\partial / \partial R), \\
H_{E N}=\sum_{i} \epsilon_{i}(R) a_{i}^{t} a_{i}+\frac{1}{2} \sum V_{i j k l}(R) a_{i}^{t} a_{j}^{t} a_{l} a_{k} \\
-\sum_{i j} \sum_{k \in f}\left[V_{i k k j}(R)\right] a_{i}^{t} a_{j}, \\
V_{i j k l}=\left\langle i j\left|e^{2}\right| r-\left.r^{\prime}\right|^{-1} \mid k l\right\rangle
\end{gathered}
$$

The $\epsilon_{i}(R)$ are the one-particle HF energies and $\mathrm{f}$ denotes the set of orbitals occupied in the HF ground state. As usually in the adiabatic approximation one could write the eigenfunctions to $\mathrm{H}$ as products $\left|x, R, \theta_{0}\right\rangle_{E} \times|R\rangle_{N}$, and further expand $\in_{i}(R), V_{i j k l}(R)$, and $U_{N N}(R, \theta)$ about $\mathrm{R}_{0}$ leaving the operators $a_{i}$ and $a_{i}^{t}$ unchanged [13]:

$$
\begin{aligned}
& H= \sum_{i} \epsilon_{i}\left(R_{0}\right) a_{i}^{t} a_{i}+\frac{1}{2} \sum V_{i j k l}\left(R_{0}\right) a_{i}^{t} a_{j}^{t} a_{l} a_{k}- \\
&-\sum_{i j} \sum_{k \in f}\left[V_{i k j k}\left(R_{0}\right)-V_{i k k j}\left(R_{0}\right)\right] a_{i}^{t} a_{j}+ \\
&+\sum_{i}\left[\sum_{s=1}^{M}\left(\frac{\partial \epsilon_{1}}{\partial R_{s}}\right)_{0}\left(R-R_{s o}\right)+\right. \\
&+ \frac{1}{2} \sum_{i} \sum_{s, s^{\prime}=1}^{M}\left(\frac{\partial^{2} \epsilon_{i}}{\partial R_{s} \partial R_{s^{\prime}}}\right)_{0}\left(R_{s}-R_{s o}\right) \times \\
&\left.\times\left(R_{s^{\prime}}-R_{s^{\prime} 0}\right)\right] a_{i}^{t} a_{i}+\ldots+U_{N N}\left(R_{0}, \theta_{0}\right)+\ldots+T_{N}\left(\frac{\partial}{\partial R}\right),
\end{aligned}
$$

where $\mathrm{M}$ is the number of normal coordinates.

Choosing $\mathrm{R}_{0}$ as the equilibrium geometry on the HF level and introducing dimensionless normal coordinates $\mathrm{Q}_{\mathrm{s}}$ one can write the following Hamiltonian (the subscript 0 stands for $\mathrm{R}_{0}$ ) [13]:

$$
\begin{gathered}
H=H_{E}+H_{N}+H_{E N}^{(1)}+H_{E N}^{(2)}, \\
H_{E}=\sum_{i} \epsilon_{i}\left(R_{0}\right) a_{i}^{t} a_{i}+\frac{1}{2} \sum V_{i j k l}\left(R_{0}\right) a_{i}^{t} a_{j}^{t} a_{l} a_{k}- \\
-\sum_{i, j} \sum_{k \in f}\left[V_{i k j k}\left(R_{0}\right)-V_{i k k j}\left(R_{0}\right)\right] a_{i}^{t} a_{j}, \\
H_{N}=\hbar \sum_{s=1}^{M} \omega_{s}\left(b_{s}^{t} b_{s}+\frac{1}{2}\right), \\
+\frac{1}{4} \sum_{i} \sum_{s, s^{\prime}=1}^{M}\left(\frac{\partial^{2} \epsilon_{i}}{\partial Q_{s} \partial Q_{s^{\prime}}}\right)_{0}^{(1)}=2^{-1 / 2} \sum_{s=1}^{M}\left(\frac{\partial \epsilon_{i}}{\partial Q_{s}}\right)_{0}\left(b_{s}+b_{s}^{t}\right)\left[a_{i}^{t} a_{i}-n_{i}\right]+ \\
\left.H_{E N}^{(2)}=2^{-3 / 2} \sum_{s^{\prime}}+b_{s^{\prime}}^{t}\right)\left[a_{i}^{t} a_{i}-n_{i}\right] \\
\times\left[\delta v_{1} a_{i}^{t} a_{j}^{t} a_{k}+\delta v_{i j k l} a_{l} a_{k} a_{i}^{t} a_{j}^{t}+2 \delta Q_{s}\right)_{0}\left(b_{s}+b_{s}^{t}\right) \times \\
+\frac{1}{8} \sum_{j}^{M} \sum_{s, s^{\prime}=1}^{M}\left(\frac{\partial^{2} V_{i j k l}}{\left.\partial Q_{s} \partial Q_{s} a_{i}^{t}\right]}\right)_{0}\left(b_{s}+b_{s}^{t}\right) \times \\
\times\left(b_{s^{\prime}}+b_{s^{\prime}}^{t}\left[\delta v_{1} a_{i}^{t} a_{j}^{t} a_{k}+\right.\right. \\
\left.+\delta v_{2} a_{l} a_{k} a_{i}^{t} a_{j}^{t}+2 \delta v_{3} a_{j}^{t} a_{k} a_{l} a_{i}^{t}\right] \\
(9)
\end{gathered}
$$




$$
\begin{gathered}
n_{i}=1 \quad i \in f, \quad \delta \sigma_{f}=1 \quad(i j k l) \in \sigma, \\
=0, \quad i \notin f, \quad=0 \quad(i j k l) \notin \sigma_{f},
\end{gathered}
$$

where the index set $\boldsymbol{v}_{1}$ means that at least $\phi_{k}$ and $\phi_{l}$ or $\phi_{i}$ and $\phi_{j}$ are unoccupied, $v_{2}$ that at most one of the orbitals is unoccupied, and $v_{3}$ that $\phi_{k}$ and $\phi_{j}$ or $\phi_{l}$ and $\phi_{j}$ are unoccupied. Besides, here for simplicity all terms leading to anharmonicities are neglected. The $\omega_{s}$ are the HF frequencies and the $b_{s}$ and $b_{s}^{t}$ are destruction and creation operators for vibrational quanta defined by

$$
\begin{gathered}
Q_{s}=(1 / \sqrt{2})\left(b_{s}+b_{s}^{t}\right), \\
\partial / \partial Q_{s}=(1 / \sqrt{2})\left(b_{s}-b_{s}^{t}\right)
\end{gathered}
$$

The first term $H_{E}$ describes the electronic motion for nuclei fixed at the HF ground state geometry. The second term $H_{N}$ describes the motion of the nuclei in the harmonic HF potential (the extension to anharmonic terms can easily be done). $H_{E N}^{(1)}$ represents the coupling of the HF particles with the nuclear motion. The coupling constants are the normal coordinate derivatives of the HF one-particle energies. The first sum in the expression for $H_{E N}^{(1)}$ is responsible for the geometry shifts and the second one for the charge of frequencies due to electrons. There is also a modification of the interaction between electrons through the coupling to the nuclear motion. The term $H_{E N}^{(2)}$, which describes this modification, is due to its nature less important than $H_{E N}^{(1)}$.

The exact solution of the one-body HF problem has been given in ref.[12]. Correspondingly in the one-particle picture the density of occupied states is given by

$$
N_{k}^{0}(\epsilon)=\frac{1}{2 \pi \hbar} \int_{-\infty}^{\infty} d t e^{i \hbar t-\varepsilon\left(t_{k}\right)}\left\langle 0\left|e^{ \pm i \hbar^{-1} \tilde{H}_{0^{t}}}\right| 0\right\rangle,
$$

with

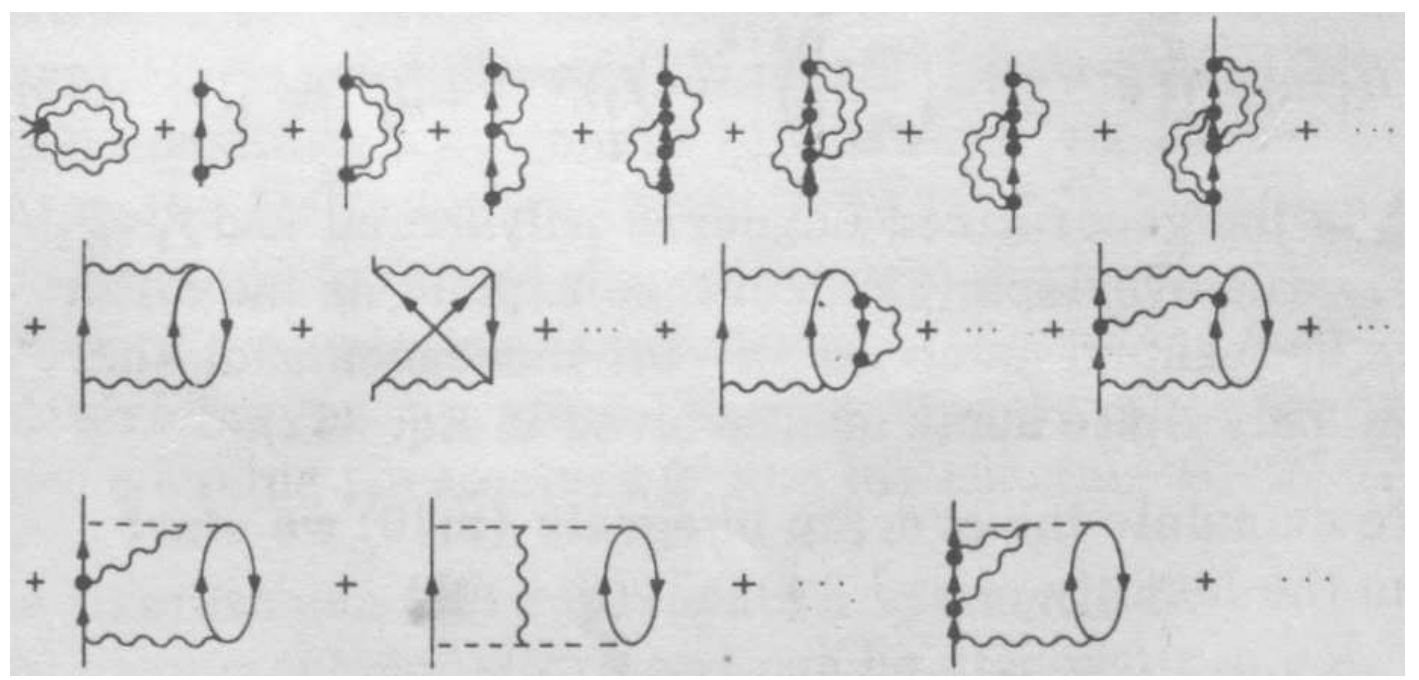

Fig. 1. The sum of diagrams contributing to the self-energy part

The one-body problem treated above results in the exact solution of the Dyson equation with the selfenergy part given by the infinite number of diagrams shown in the first row of Fig. 1 and the corresponding Green's function is as follows [12-14]:

$$
\begin{gathered}
\tilde{H}_{0}=\sum_{s=1}^{M} \hbar \omega_{s} b_{s}^{t} b_{s}+\sum_{s=1}^{M} g_{s}^{k}\left(b_{s}+b_{s}^{t}\right)+ \\
\sum_{s, s^{\prime}=1}^{M} \gamma_{s s^{\prime}}^{k}\left(b_{s}+b_{s}^{t}\right)\left(b_{s^{\prime}}+b_{s^{\prime}}^{t}\right) \\
g_{s}^{i}= \pm \frac{1}{\sqrt{2}}\left(\frac{\partial \epsilon_{i}}{\partial Q_{s}}\right)_{0}, \quad \gamma_{s s^{\prime}}^{i}= \pm \frac{1}{4}\left(\frac{\partial^{2} \epsilon_{i}}{\partial Q_{s} \partial Q_{s^{\prime}}}\right)_{0} .
\end{gathered}
$$

In a diagrammatic method in order to obtain the function $N_{k}(\epsilon)$ one should calculate the Green's function $G_{k k^{\prime}}(\epsilon)$ first:

$$
\begin{gathered}
G_{k k^{\prime}}(\epsilon)=-i \hbar^{-1} \int_{-\infty}^{\infty} d t e^{i \hbar^{-1} \epsilon t} \times \\
\times\left\langle\psi_{0}\left|T\left\{a_{k}(t) a_{k}^{t}(0)\right\}\right| \psi_{0}\right\rangle
\end{gathered}
$$

where $\boldsymbol{T}$ is Wick's time ordering operator and the function $\mathrm{E}_{k}(\mathrm{)})$ then follows from the relation

$$
\begin{aligned}
\pi N_{k}(\epsilon) & =a \operatorname{Im} G_{k k}(\epsilon-a i \eta), \\
a & =- \text { signe }_{k},
\end{aligned}
$$

where $\eta$ is a positive infinitesimal. Choosing the unperturbed Hamiltonian $H_{0}$ to be

$$
H_{0}=\sum \epsilon_{i} a_{i}^{t} a_{i}+H_{N}
$$

one finds for the corresponding Green's functions

$$
G_{k k}^{0}(\epsilon)=\delta_{k k^{\prime}} /\left(\epsilon-\epsilon_{k}-a i \eta\right)
$$

The Dyson equation

$$
G_{k k^{\prime}}=G_{k k^{\prime}}^{0}+\sum_{k^{\prime \prime}} G_{k k^{0}}^{0} \Sigma_{k k^{\prime \prime}} G_{k^{\prime \prime} k^{\prime}}
$$

relates the Green's functions to the free ones introducing a new function $\Sigma_{k k^{\prime \prime}}(\epsilon)$ called the (proper) self-energy part. In in order to calculate $\Sigma_{k k^{\prime}}$, a well-known diagrammatic method is used. The sum of Feynman diagrams leading to the self-energy part is shown in Fig. 1. All notations are standard. 

lows:

The corresponding Dyson-like equation is as fol-

$$
G_{k k^{\prime}}(\epsilon)=G_{k k^{\prime}}^{O B}(\in)+\sum_{k k^{\prime}} G_{k k}^{O B}(\in) \Phi_{k k^{\prime \prime}} G_{k^{\prime \prime} k^{\prime}}(\in)
$$

where $\Phi_{k k^{\prime}}$, is equal to $\Sigma_{k k^{\prime}}$, less the diagrams of the first row in Fig.1. The perturbation expansion of $\Phi$ is shown in Fig. 2 where $i G_{k k^{\prime}}^{O B}$, is symbolized by a double solid line.

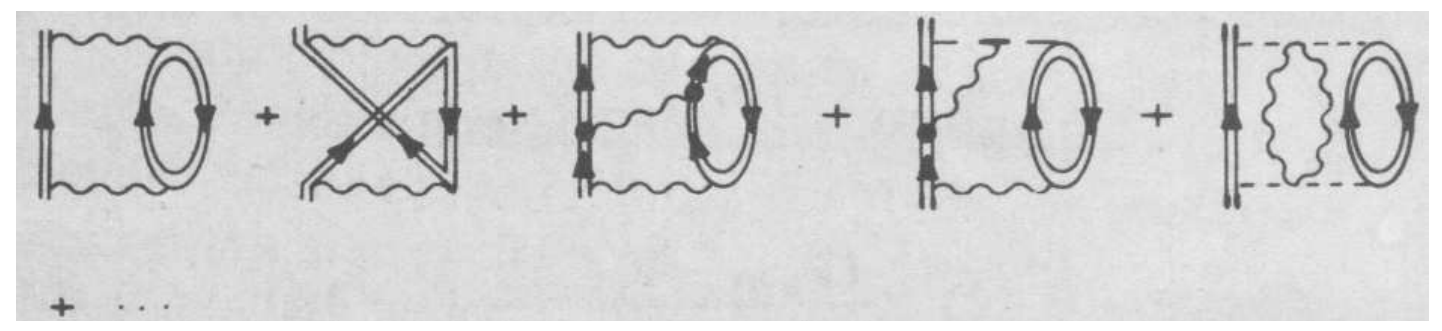

Fig. 2. Perturbation expansion of $\Phi_{\mathrm{kk}}$

The expression for a sum of the first 2 diagrams appearing in Fig. 2 are written by a standard way:

$$
\begin{gathered}
\Phi_{k k^{\prime}}(\in)=\sum_{\substack{i, j \in F \\
l \notin F}} \sum_{n_{i}, n_{j}, n_{l}} \frac{\left(V_{k l i j}-V_{k l i j}\right) V_{k^{\prime} l j} U_{n_{i}} U_{n_{j} j} U_{n_{l} l}}{\epsilon+E_{l}-E_{i}-E_{j}}+ \\
\quad+\sum_{\substack{i, j \in F \\
l \notin F}} \sum_{n_{i}, n_{j}, n_{l}} \frac{\left(V_{k l i j}-V_{k l i j}\right) V_{k^{\prime} l j} U_{n_{i}} U_{n_{j} j} U_{n_{l} l}}{\epsilon+E_{l}-E_{i}-E_{j}}
\end{gathered}
$$

where

$$
U_{n, i=}\left|\left\langle\hat{n}_{i}\left|U_{i}\right| 0\right\rangle\right|^{2} \text { and } E_{i}=\epsilon_{i} \mp \Delta \epsilon_{i} \mp h \hat{n}_{i} \cdot \varpi_{i}
$$

The direct method for calculation of $N_{k}(\in)$ as the imaginary part of the corresponding Green's function implicitly includes the determination of the V. I. P. s of the reference molecule and then of $N_{k}(\in)$. The zeros of the functions

$$
D_{k}(\epsilon)=\in-\left[\epsilon^{o p}+\Sigma(\epsilon)\right]_{k},
$$

where $\left(\epsilon^{o p}+\Sigma\right)$ denotes the $\boldsymbol{k}$ th eigenvalue of the diagonal matrix of the one-particle energies added to the matrix of the self-energy part, are the negative V. I. P. 's for a given geometry. Further it is easily to write [12]:

$$
\begin{gathered}
(\text { V.I.P. })_{k}=-\left(\epsilon_{k}+F_{k}\right), \\
F_{k}=\Sigma_{k k}\left(-(\text { V.I.P. })_{k}\right) \approx \frac{1}{1-\partial \Sigma_{k k}\left(\epsilon_{k}\right) / \partial_{\epsilon}} \Sigma_{k k}\left(\epsilon_{k}\right) .
\end{gathered}
$$

Expanding the ionic energy $E_{k}^{N-1}$ about the equilibrium geometry of the reference molecule in a power series of the normal coordinates of this molecule:

$$
E_{k}^{N-1}=E_{k}^{N-1}(0)-\sum_{s=1}^{M}\left(\frac{\partial\left(\epsilon_{k}+F_{k}\right)}{\partial Q_{s}}\right)_{0} Q_{s}-
$$

$$
-\frac{1}{2 !} \sum_{s, s^{\prime}=1}^{M}\left(\frac{\partial^{2}\left(\epsilon_{k}+F_{k}-E_{0}^{N}\right)}{\partial Q_{s} \partial Q_{s^{\prime}}}\right)_{0} Q_{s} Q_{s^{\prime}}
$$

leads to a set of linear equations in the unknown normal coordinate shifts $\Delta \boldsymbol{Q}_{s}$,

$$
\begin{gathered}
-\left(\frac{\partial\left(\epsilon_{k}+F_{k}\right)}{\partial Q_{s}}\right)_{0}=\sum_{s^{\prime} \neq s}\left(\frac{\partial^{2}\left(\epsilon_{k}+F_{k}\right)}{\partial Q_{s} \partial Q_{s^{\prime}}}\right)_{0} \delta Q_{s^{\prime}}, \\
+\left[\left(\frac{\partial^{2}\left(\epsilon_{k}+F_{k}\right)}{\partial Q_{3}^{2}}\right)_{0}-h \omega_{s}\right] \delta Q_{s}, s=1 \ldots M,
\end{gathered}
$$

where $\omega_{s}$ are frequencies of the reference molecule. The new coupling constants are then:

$$
\begin{gathered}
g_{1}= \pm(1 / \sqrt{2})\left[\partial\left(\epsilon_{k}+F_{k}\right) / \partial Q_{l}\right]_{0} \\
\gamma_{l^{\prime}}= \pm\left(\frac{1}{4}\right)\left[\partial^{2}\left(\epsilon_{k}+F_{k}\right) / \partial Q_{l} / \partial Q_{l^{\prime}}\right]_{0}
\end{gathered}
$$

Thee coupling constants $g_{l}$ and $y_{l l^{\prime}}$ are calculated by the well-known perturbation expansion of the selfenergy part using the Hamiltonian $\boldsymbol{H}_{E N}$ of Eq. (6). In second order one obtains:

$$
\begin{aligned}
& \sum_{k k}^{(2)}(\epsilon)=\sum_{\substack{i, j \\
s \notin F}} \frac{\left(V_{k s i j}-V_{k s j i}\right) V_{k s i j}}{\epsilon+\epsilon_{s}-\epsilon_{i}-\epsilon_{j}}+ \\
& +\sum_{\substack{i, j \\
s \in F}} \frac{\left(V_{k s i j}-V_{k s j i}\right) V_{k s i j}}{\epsilon+\epsilon_{s}-\epsilon_{i}-\epsilon_{j}}
\end{aligned}
$$

and the coupling constant $g_{p}$ can be written as

$$
g_{l} \approx \pm \frac{1}{\sqrt{2}} \frac{\partial \in_{k}}{\partial Q_{l}} \frac{1+q_{k}(\partial / \partial \in) \sum_{k k}\left[-(\text { V.I.P. })_{k}\right]}{1-(\partial / \partial \in) \sum_{k k}\left[-(\text { V.I.P. })_{k}\right]},
$$

where

$$
q_{k}=\frac{\sum \frac{\left(V_{k s i j}-V_{k s j i}\right)^{2}}{\left[-(\text { V.I.P. })_{k}+\epsilon_{s}-\epsilon_{i}-\epsilon_{j}\right]^{2}}\left[\frac{\partial \epsilon_{s}}{\partial Q_{l}}-\frac{\partial \epsilon_{i}}{\partial Q_{l}}-\frac{\partial \epsilon_{j}}{\partial Q_{l}}\right]}{\frac{\partial \epsilon_{k}}{\partial Q_{l}} \sum \frac{\left(V_{k s i j}-V_{k s j i}\right)^{2}}{\left[-(\text { V.I.P. })_{k}+\epsilon_{s}-\epsilon_{i}-\epsilon_{j}\right]^{2}}}
$$


It is suitable to use further the pole strength of the corresponding Green's function and then

$$
\rho_{k}=\left\{1-\frac{\partial}{\partial \epsilon} \sum_{k k}\left[-(\text { V.I.P. })_{k}\right]\right\}^{-1} ; 1 \geq \rho_{k} \geq 0,
$$

$$
g_{l} \approx g_{l}^{0}\left[\rho_{k}+q_{k}\left(\rho_{k}-1\right)\right], g_{l}^{0}= \pm 2^{-1 / 2} \partial \in_{k} / \partial Q_{l}
$$

Below we firstly give the DFT definition of the pole strength corresponding to V. I. P.'s and confirm earlier data $[13,14]: \boldsymbol{p}_{\boldsymbol{k}} \approx 0,8-0,95$. The closeness of $\boldsymbol{p}_{\boldsymbol{k}}$ to 1 in fact means that a role of the multi-body correlation effects is small $\left(g_{l} \approx g_{l}^{0}\right)$. The above presented results can be usefully treated in the terms of the correlation and reorganization effects. Usually it is introduced the following expression for an I.P.:

$$
\begin{gathered}
(I . P .)_{k}=-\epsilon_{k}-\sum_{j \notin, i \in F} \frac{\left(V_{k i k j}-V_{k i j k}\right)}{\epsilon_{j}-\epsilon_{i}}- \\
-\frac{1}{2} \sum_{\substack{i \in F \\
j, i \notin F}} \frac{\left(V_{k j i l}-V_{k i j}\right)}{\epsilon_{k}+\epsilon_{i}-\epsilon_{j}-\epsilon_{l}}\left(1-\delta_{i k}\right) \\
-\frac{1}{2} \sum_{\substack{p, q \in F \\
j \notin F F}} \frac{\left(V_{k j p q}-V_{k j q p}\right)^{2}}{\epsilon_{k}+\epsilon_{i}-\epsilon_{p}-\epsilon_{q}}\left(1-\delta_{k p}\right)\left(1-\delta_{k q}\right)
\end{gathered}
$$

The first correction term is due to reorganization, the remaining correction terms are due to correlation effects. Then the coupling constant $g_{p}$ can be written as

$$
\begin{gathered}
g_{l} \approx g_{l}^{0}\left\{1+\sum_{j \notin F} \frac{\left(V_{k k k j}\right)^{2}}{\left(\epsilon_{j}-\epsilon_{k}\right)^{2}}-\right. \\
-\frac{1}{2}\left[\sum_{\substack{i \in F \\
j, l \notin F}} \frac{\left(V_{k i j l}-V_{k i l j}\right)^{2}}{\left(\epsilon_{k}+\epsilon_{i}-\epsilon_{j}-\epsilon_{l}\right)^{2}}\left(1-\delta_{k i}\right)+\right. \\
\left.\left.+\sum_{\substack{p, q \in F \\
j, \notin F}} \frac{\left(V_{k j p q}-V_{k j q p}\right)^{2}}{\left(\epsilon_{k}+\epsilon_{i}-\epsilon_{p}-\epsilon_{q}\right)^{2}}\left(1-\delta_{k q}\right)\left(1-\delta_{k p}\right)\right]\right\}
\end{gathered}
$$

The second coupling constant can be written

$$
\gamma_{l l}=\gamma_{l l}^{0}\left(\frac{g_{l}}{g_{l}^{0}}\right)+\frac{1}{4} \sqrt{2} g_{l}^{0} \frac{\partial}{\partial Q_{l}}\left(\frac{g_{l}}{g_{l}^{0}}\right)
$$

$\gamma_{l l}^{0}$, is defined analogously $g_{l}^{0}$.

\section{QUASIPARTICLE DENSITY FUNCTIONAL THEORY}

The quasiparticle Fermi-liquid version of the DFT theory has been presented in ref. [11,14] (see also [18$23,25]$ ), starting from the problem of searching for the optimal one-electron representation [2-5]. One of the simplified recipes represents the Kohn-Sham DFT theory [15]. Earlier new QED DFT version, based on the formally exact QED perturbation theory (energy approach), has been developed and a new approach to construction of the optimized one-quasiparticle representation has been proposed (look details in ref.
[11]). The energy approach uses the adiabatic GellMann and Low formula for the energy shift $\Delta \boldsymbol{E}$ with electrodynamic scattering matrice. In a modern theory of molecules there is a number of tasks, where an accurate account for the complex exchange-correlation effects, including the continuum pressure, energy dependence of a mass operator etc., is critically important. It includes also the calculation of the vibration structure for the molecular systems. In this case it can be very useful the quasiparticle DFT $[11,14]$.

In order to get the master equations and construct an optimal basis of the one-particle wave functions $\varphi_{\lambda}$ one could use the Green's function method. Let us define the one-particle Hamiltonian for functions $\varphi_{\lambda}$ so that the Greens' function pole part in the $\left(\varphi_{\lambda}\right.$ ) representation is diagonal on $\lambda$. Starting equation is the Dyson equation for multi-electron (for example atom or molecule):

$$
\begin{gathered}
\left(\varepsilon-p^{2} / 2+\sum Z_{\alpha} / r_{\alpha}\right) \cdot G\left(x, x^{\prime}, \varepsilon\right)- \\
-\int d x^{\prime} \sum\left(x, x^{\prime}, \varepsilon\right)=\delta\left(x-x^{\prime}\right)
\end{gathered}
$$

where $x=(r, s)$ are the spatial and spin variables, $\sum$ is the mass operator; $\mathrm{Z}$, as usually, a charge of a nucleus (nuclei) " $\alpha$ ", $G$ is the Green's function. In the representation of auxiliary functions $\varphi_{\lambda}^{\prime}$ the equation (67) has the following form:

$$
\left(\varepsilon \cdot \delta_{\lambda \lambda_{1}}-\left[\frac{p^{2}}{2}-\sum \frac{Z_{\alpha}}{r_{\alpha}}+\sum\left(x, x^{\prime}, \varepsilon\right)\right]_{\lambda \lambda_{1}}\right) G_{\lambda \lambda^{\prime}}=\delta_{\lambda \lambda^{\prime}}
$$

where $\lambda_{1}$ is an index of summation. It is natural to choose $\varphi_{\lambda}$ so that the following expression will be diagonal:

$$
\left[p^{2} / 2-\sum Z_{\alpha} / r_{\alpha}+\sum\left(x, x^{\prime}, \varepsilon\right)\right]_{\lambda \lambda_{1}}=E_{\lambda}(\varepsilon) \cdot \delta_{\lambda \lambda_{1}}
$$

Then the Green's function is diagonal on $\lambda$ :

$$
G_{\lambda \lambda^{\prime}}=G_{\lambda} \cdot \delta_{\lambda \lambda^{\prime}}, G_{\lambda}=1 /\left[\varepsilon-E_{\lambda}(\varepsilon)\right]
$$

and the functions $\varphi_{\lambda}^{\prime}$, which diagonalizes $\mathrm{G}$, satisfy to equation as follows: :

$$
\begin{gathered}
\left(p^{2} / 2-\sum_{\alpha} Z_{\alpha} / r_{\alpha}\right) \varphi_{\lambda}^{\prime}(x, \varepsilon)+ \\
+\int \sum\left(x, x^{\prime}, \varepsilon\right) \varphi_{\lambda}^{\prime}\left(x_{1}, \varepsilon\right) d x_{1}-E_{\lambda}(\varepsilon) \varphi_{\lambda}^{\prime}(x, \varepsilon)
\end{gathered}
$$

One could introduce the mixed representation for a mass operator as follows:

$$
\sum(x, p, \varepsilon)=\int \sum\left(x, x_{1}, \varepsilon\right) \exp \left[i\left(r-r_{1}\right) p\right] d r_{1}
$$

Then equation (37) with account for of the expression (38) can be written as follows:

$$
\begin{gathered}
{\left[p^{2} / 2-\sum_{\alpha} Z_{\alpha} / r_{\alpha}+\sum(x, p, \varepsilon)\right] \varphi_{\lambda}^{\prime}(x, \varepsilon)=} \\
=E_{\lambda}(\varepsilon) \varphi_{\lambda}^{\prime}(x, \varepsilon)
\end{gathered}
$$

It can be shown that an operator $p=i v$ in (33) acts on functions which are on the right of $\sum(x, p, \varepsilon)$. So, in order to find the one-particle energies, defined by the pole part of the Green's function $G$, it is sufficient to know the functions $\varphi_{\lambda}^{\prime}$ under $\varepsilon=\varepsilon_{\lambda}$. The Greens' function pole part is as follows:

$$
G_{\lambda \lambda^{\prime}}=a^{\lambda} \delta_{\lambda \lambda^{\prime}} /\left(\varepsilon-\varepsilon_{\lambda}+i \gamma_{\lambda}\right)
$$


where

$$
\begin{gathered}
a^{\lambda}=1 /\left.\left(1-\partial E_{\lambda} / \delta \varepsilon\right)\right|_{\varepsilon=\varepsilon_{\lambda}},\left.(\partial E / \partial \varepsilon)\right|_{\varepsilon=\varepsilon_{\lambda}}=\left.(\partial E / \partial \varepsilon)\right|_{\lambda \lambda} \\
\varepsilon_{\lambda}=E_{\lambda}(\varepsilon)=\left.\left\{p^{2} / 2-\sum_{\alpha} Z_{\alpha} / r_{\alpha}+\sum(x, p, \varepsilon)\right\}\right|_{\lambda \lambda}
\end{gathered}
$$

The functions $\varphi_{\lambda}^{\prime}(x)=\varphi_{\lambda}^{\prime}\left(x, \varepsilon_{\lambda}\right)$ are satisfying to following equation:

$$
\left[p^{2} / 2-\sum_{\alpha} Z_{\alpha} / r_{\alpha}+\sum\left(x, p, \varepsilon_{\lambda}\right)\right] \varphi_{\lambda}=\varepsilon_{\lambda} \varphi_{\lambda}(x)
$$

Introducing an expansion for self-energy part $\sum$ into set on degrees $x, \varepsilon-\varepsilon_{F}, p^{2}-p_{F}^{2}$ (here $\varepsilon_{F}$ and $p_{F}$ are the Fermi energy and pulse correspondingly):

$$
\begin{gathered}
\sum(x, p, \varepsilon)=\sum_{0}(x)+ \\
+\left(\partial \sum / \partial p^{2}\right)\left(p^{2}-p_{F}^{2}\right)+\left(\partial \sum / \partial \varepsilon\right)\left(\varepsilon-\varepsilon_{F}\right)+\ldots
\end{gathered}
$$

then equation (42) is rewritten as follows:

$$
\begin{gathered}
{\left[p^{2} / 2-\sum_{\alpha} Z_{\alpha} / r_{\alpha}+\sum_{0}(x)+\right.} \\
\left.+p\left(\partial \sum / \partial p^{2}\right) p\right] \Phi_{\lambda}(x)=\left(1-\partial \sum / \partial \varepsilon\right) \varepsilon_{\lambda} \Phi_{\lambda}(x)
\end{gathered}
$$

The functions $\Phi_{\lambda}$ in (77) are orthogonal with a weight $\rho_{k}^{-1}=a^{-1}=\left[1-\partial \sum / \partial \varepsilon\right]$. Now one can introduce the wave functions of the quasiparticles $\varphi_{\lambda}=a^{-1 / 2} \Phi_{\lambda}$, which are, as usually, orthogonal with weight 1. For complete definition of $\left\{\varphi_{2}\right\}$ it should be determined the values $\sum_{0}, \partial \sum / \partial p^{2}, \partial \sum / \partial \varepsilon$. Naturally, the equations (43) can be obtained on the basis of the variational principle, if we start from a Lagrangian of a system $L_{q}$ (density functional). It should be defined as a functional of the following quasiparticle densities:

$$
\begin{aligned}
& v_{0}(r)=\sum_{\lambda} n_{\lambda}\left|\Phi_{\lambda}(r)\right|^{2}, \\
& v_{1}(r)=\sum_{\lambda} n_{\lambda}\left|\nabla \Phi_{\lambda}(r)\right|^{2}, \\
& v_{2}(r)=\sum_{\lambda} n_{\lambda}\left[\Phi_{\lambda}^{*} \Phi_{\lambda}-\Phi_{\lambda}^{*} \Phi_{\lambda}\right] .
\end{aligned}
$$

The densities $v_{0}$ and $v_{1}$ are similar to the HF electron density $\rho(\rho=v \cdot a)$ and kinetical energy density correspondingly; the density $v_{2}$ has no an analog in the HF or standard Kohn-Sham theory and appears as result of account for the energy dependence of the mass operator. A Lagrangian $L_{q}$ can be written as a sum of a free Lagrangian and Lagrangian of interaction:

$$
L_{q}=L_{q}^{0}+L_{q}^{\mathrm{int}}
$$

where a free Lagrangian $L_{q}^{0}$ has a standard form:

$$
L_{q}^{0}=\int d r \sum_{\lambda} n_{\lambda} \Phi_{\lambda}^{*}\left(i \partial / \partial t-\varepsilon_{p}\right) \Phi_{\lambda},
$$

And an interaction Lagrangian is defined in the form, which is characteristic for a standard KohnSham DFT theory (as a sum of the Coulomb and exchange-correlation terms), however, it takes into account for the energy dependence of a mass operator:

$$
L_{q}^{\mathrm{int}}=L_{K}-\frac{1}{2} \sum_{i, k=0}^{2} \int \beta_{i k} F\left(r_{1}, r_{2}\right) v_{i}\left(r_{1}\right) v_{k}\left(r_{2}\right) d r_{1} d r_{2}
$$

where $\beta_{i k}$ are some constants (look below), $\mathrm{F}$ is an effective potential of the exchange-correlation interaction. Let us explain here the essence of the introduced constants. Indeed, in some degree they have the same essence as the similar constants in the well-known Landau Fermi-liquid theory and Migdal finite Fermi-systems theory. The Coulomb interaction part $L_{K}$ looks as follows:

$$
\begin{aligned}
& L_{K}=-\frac{1}{2} \int\left[1-\sum_{2}\left(r_{1}\right)\right] v_{0}\left(r_{1}\right)[1- \\
& \left.-\sum_{2}\left(r_{2}\right)\right] v_{0}\left(r_{2}\right) /\left|r_{1}-r_{2}\right| d r_{1} d r_{2}
\end{aligned}
$$

where $\sum_{2}=\partial \sum / \partial \varepsilon$. Regarding the exchange-correlation potential $\boldsymbol{F}$, it should be noted the there are many possible approximations (directly in the density -functional theory and its modern generalizations). One of the suitable forms for this potential is the Ivanov-Ivanova potential (look details in ref. [11]):

$$
\begin{gathered}
F\left(r_{1}, r_{2}\right)=X\left(\int d r \rho_{c}^{(0) 1 / 3}(r) /\left|r-r_{1}\right|\left|r-r_{2}\right|-\right. \\
-\left(\int d r^{\prime} \rho_{c}^{(0) 1 / 3}\left(r^{\prime}\right) /\left|r_{1}-r^{\prime}\right| \cdot\right. \\
\left.\cdot \int d r^{\prime \prime} \rho_{c}^{(0) 1 / 3}\left(r^{\prime \prime}\right) /\left|r^{\prime \prime}-r_{2}\right|\right) / \int d r \rho_{c}^{(0) 1 / 3}(r)
\end{gathered}
$$

where $\mathrm{X}$ is the numerical coefficient. It has been obtained on the basis of calculating the RayleighSchrцdinger perturbation theory Feynman diagrams of the second and higher order (so called polarization diagrams) in the Thomas-Fermi approximation [26]. The relativistic generalization of the potential (48) is obtained in ref.[11].

In the local density approximation in the density functional the potential $\boldsymbol{F}$ can be expressed through the exchange-correlation pseudo-potential $V_{X C}$ as follows $[15$,$] :$

$$
F\left(r_{1}, r_{2}\right)=\delta V_{X C} / \delta v_{0} \cdot \delta\left(r_{1}-r_{2}\right) .
$$

Further, one can get the following expressions for $\sum_{i}=-\delta L_{q}^{\mathrm{int}} / \delta v_{1}$ :

$$
\begin{gathered}
\sum_{0}=\left(1-\sum_{e}\right) V_{K}+\sum_{0}^{e x}+ \\
+\frac{1}{2} \beta_{00} \delta^{2} V_{X C} / \delta v^{2} \cdot v_{0}^{2}+\beta_{00} \delta V_{X C} / \delta v_{0} \cdot v_{0}+ \\
+\beta_{01} \delta V_{X C} / \delta v_{0} \cdot v_{1}+\beta_{01} \delta^{2} V_{X C} / \delta v_{0}^{2} \cdot v_{0} v_{1}+ \\
+\beta_{02} \delta^{2} V_{X C} / \delta v_{0}^{2} \cdot v_{0} v_{2}+\beta_{02} \delta V_{X C} / \delta v_{0} \cdot v_{2} \\
\sum_{1}=\beta_{01} \delta V_{X C} / \delta v_{0} \cdot v_{0}+ \\
+\beta_{12} \delta V_{X C} / \delta v_{0} \cdot v_{2}+\beta_{11} \delta V_{X C} / \delta v_{0} \cdot v_{1} \\
\sum_{2}=\beta_{02} \delta V_{X C} / \delta v_{0} \cdot v_{0}+ \\
+\beta_{12} \delta V_{X C} / \delta v_{0} \cdot v_{1}+\beta_{22} \delta V_{X C} / \delta v_{0} \cdot v_{2}
\end{gathered}
$$

Here $V_{K}$ is the Coulomb term (look above), $\sum_{0}^{e x}$ is the exchange term. Using the known canonical relationship $H_{q}=\Phi_{\lambda}^{*} \delta L_{q} / \delta \Phi_{\lambda}^{*}+\Phi_{\lambda} \delta L_{q} / \delta \Phi_{\lambda}-L_{q}$ after some transformations one can receive the expression for the quasiparticle Hamiltonian, which is corresponding to a Lagrangian $L_{q}$ :

$$
\begin{gathered}
H_{q}=H_{q}^{0}+H_{q}^{\mathrm{int}}=H_{q}^{0}-L_{K}+\frac{1}{2} \beta_{00} \delta V_{X C} / \delta v_{0} \cdot v_{0}^{2}+ \\
+\beta_{01} \delta V_{X C} / \delta v_{0} \cdot v_{0} \cdot v_{1}+\frac{1}{2} \beta_{11} \delta V_{X C} / \delta v_{0} \cdot v_{1}^{2}- \\
-\frac{1}{2} \beta_{22} \delta V_{X C} / \delta v_{0} \cdot v_{2}^{2}
\end{gathered}
$$


In further applications as potential $V_{X C}$ it is suitable to use the exchange-correlation pseudo-potential which contains the correlation (Gunnarsson-Lundqvist) potential and exchanger Kohn-Sham one [11]:

$$
V_{X C}(r)=f(\theta) V_{X}(r)-0,0333 \cdot \ln \left[1+18,376 \cdot \rho^{1 / 3}(r)\right]
$$

where

$$
V_{X}=-(1 / \pi)\left[3 \pi^{2} \cdot \rho(r)\right]^{1 / 3}
$$

is the Kohn-Sham exchange potential, $\theta=\left[3 \pi^{2} \rho\right]^{1 / 3} / c$, and function $f(\theta)$ is some function. Using the above written formula, one can simply define the values (46), (50).

Further let us give the corresponding comments regarding the constants $\beta_{i k}$. First of all, it is obvious that the terms with constants $\beta_{01}, \beta_{11}, \beta_{12}, \beta_{22}$ give omitted contribution to the energy functional (at least in the zeroth approximation in comparison with others), so they can be equal to zero. The value for a constant $\beta_{00}$ in some degree is dependent upon the definition of the potential $V_{X C}$. If as $V_{X C}$ it is use one of the correct exchange-correlation potentials from the standard density functional theory, then without losing a community of statement, the constant $\beta_{00}$ can be equal to 1 . The constant $\beta_{02}$ can be in principle calculated by analytical way, but it is very useful to remember its connection with a spectroscopic factor $F_{s p}$ of the molecular system (it is usually defined from the ionization cross-sections) $[11,14]$ :

$$
F_{s p}=\left\{1-\frac{\partial}{\partial \epsilon} \sum_{k k}\left[-(\text { V.I.P. })_{k}\right]\right\}
$$

The term $\partial \sum / \partial \varepsilon$ is defined above. It is easily to understand the this definition is in fact corresponding to the pole strength of the corresponding Green's function. It is interesting to discuss the possible analogous universality of $\beta_{i k}$ and the constants in the well-known Landau Fermi-liquid theory and Migdal finite Fermi-systems theory. Indeed, as we know now, the entire universality of the constants in the last theories is absent, though a range of its changing is quite little. Without a detailed explanation, we note here that the corresponding constants in our theory possess the same universality as ones in the Landau Fermi-liquid theory and Migdal finite Fermi-systems theory. More detailed explanation requires a careful check. Further it is obvious that omitting the energy dependence of the mass operator (i.e. supposing $\beta_{02}=0$ ) the quasiparticle density functional theory can be resulted in the standard Kohn-Sham theory. In this essence presented approach to definition of the functions basis $\left\{\Phi_{\lambda}\right\}$ of Hamiltonian $H_{q}$ can be treated as an improved in comparison with similar basise's of other one-particle representations (HF, Hatree-Fock-Slater, Kohn-Sham etc.). Naturally, this advancement can be manifested during studying those properties of the multi-electron systems, when an accurate account for the complex exchange-correlation effects, including a continuum pressure, energy dependence of a mass operator etc., is critically important.

\section{APPLICATION OF THE COMBINED METHOD TO DIATOMICS PHOTOELECTRON SPECTRA}

As an object of studying we choose the diatomic molecule of $\mathrm{N}_{2}$ for application of the combined Green's function method and quasiparticle DFT approach. The nitrogen molecule has been naturally discussed in many papers. The valence V. I. P. 's of $\mathrm{N}_{2}$ have been calculated $[1,13,14,24]$ by the method of Green's functions and therefore the pole strengths $\boldsymbol{p}_{k}$ are known and the mean values $\boldsymbol{q}_{k}$ can be estimated. It should be reminded that the $\mathrm{N}_{2}$ molecule is the classical example where the known Koopmans' theorem even fails in reproducing the sequence of the V. I. P. 's in the PE spectrum. From the HF calculation of Cade et al.[24] one finds that including reorganization the V. I. P. 's assigned by $\sigma_{g}$ and $\sigma_{u}$ improve while for $\pi \mathrm{V}$. I. P. the good agreement between the Koopmans value and the experimental one is lost, leading to the same sequence as given by Koopmans' theorem. Earlier $[13,14]$ it has been shown that the nitrogen spectra can be in principle reproduced by applying a one-particle theory with account of the correlation and reorganization effects. The above-mentioned Green's functions calculation which takes account of reorganization and correlation effects leads to the experimental sequence of V. I. P.'s. In Table 1 the experimental V. I. P. 's (a), the one-particle HF energies (b), the V. I. P. 's calculated by Koopmans' theorem plus the contribution of reorganization (c), the V. I. P. 's calculated with Green's functions method (d), the combined Green functions and DFT approach (e) and the corresponding pole strengths $(\mathrm{d}, \mathrm{e})$ are listed.

Table 1

The experimental and calculated V. I. P.'s (in $\mathrm{eV}$ ) of $\mathrm{N}_{2} \cdot R_{k}$ is the contribution of reorganization; $p_{k}$ stands for pole strength

\begin{tabular}{|c|c|c|c|c|c|c|c|}
\hline $\begin{array}{c}\text { Or- } \\
\text { bital }\end{array}$ & $\begin{array}{c}\text { Exptl } \\
\text { V.I.P.s }\end{array}$ & $-\epsilon_{k}^{b}$ & $\begin{array}{c}-\left(\epsilon_{k}+\right. \\
\left.+R_{k}\right)^{c}\end{array}$ & $\begin{array}{c}\text { Calc } \\
\text { V.I.P.s }\end{array}$ & $\rho_{k}^{d}$ & $\begin{array}{c}\text { Calce } \\
\text { V.I.P.s }\end{array}$ & $\rho_{k}^{e}$ \\
\hline $3 \sigma_{g}$ & 15,60 & 17,36 & 16,01 & 15,50 & 0,91 & 15,52 & 0,913 \\
\hline $1 \pi_{u}$ & 16,98 & 17,10 & 15,67 & 16,83 & 0,94 & 16,85 & 0,942 \\
\hline $2 \sigma_{u}$ & 18,78 & 20,92 & 19,93 & 18,59 & 0,87 & 18,63 & 0,885 \\
\hline
\end{tabular}

Analysis shows that the data, obtained within the standard Green functions approach and combined method are very much close. Taking into account a simplification of the method scheme within the DFT approach, the standard Green's function theory ( in particular the Cederbaum-Domske theory [12]) looks more attractive else. As it is known, of the three bands in the experimental low-energy spectrum of the $\mathrm{N}_{2}$ molecule ( Fig. 3), only the $1 \pi_{u}$ band exhibits a strong vibrational structure.

When the change of frequency due to ionization is small, the density of states can be well approximated using only one parameter $g$ :

$$
\begin{gathered}
N_{k}(\in)=\sum_{n=0}^{\infty} e^{-s} \frac{S^{n}}{n !} \delta\left(\in-\epsilon_{k}+\Delta \epsilon_{k}+n \cdot \hbar \omega\right) \\
S=g^{2}(\hbar \omega)^{-2}
\end{gathered}
$$




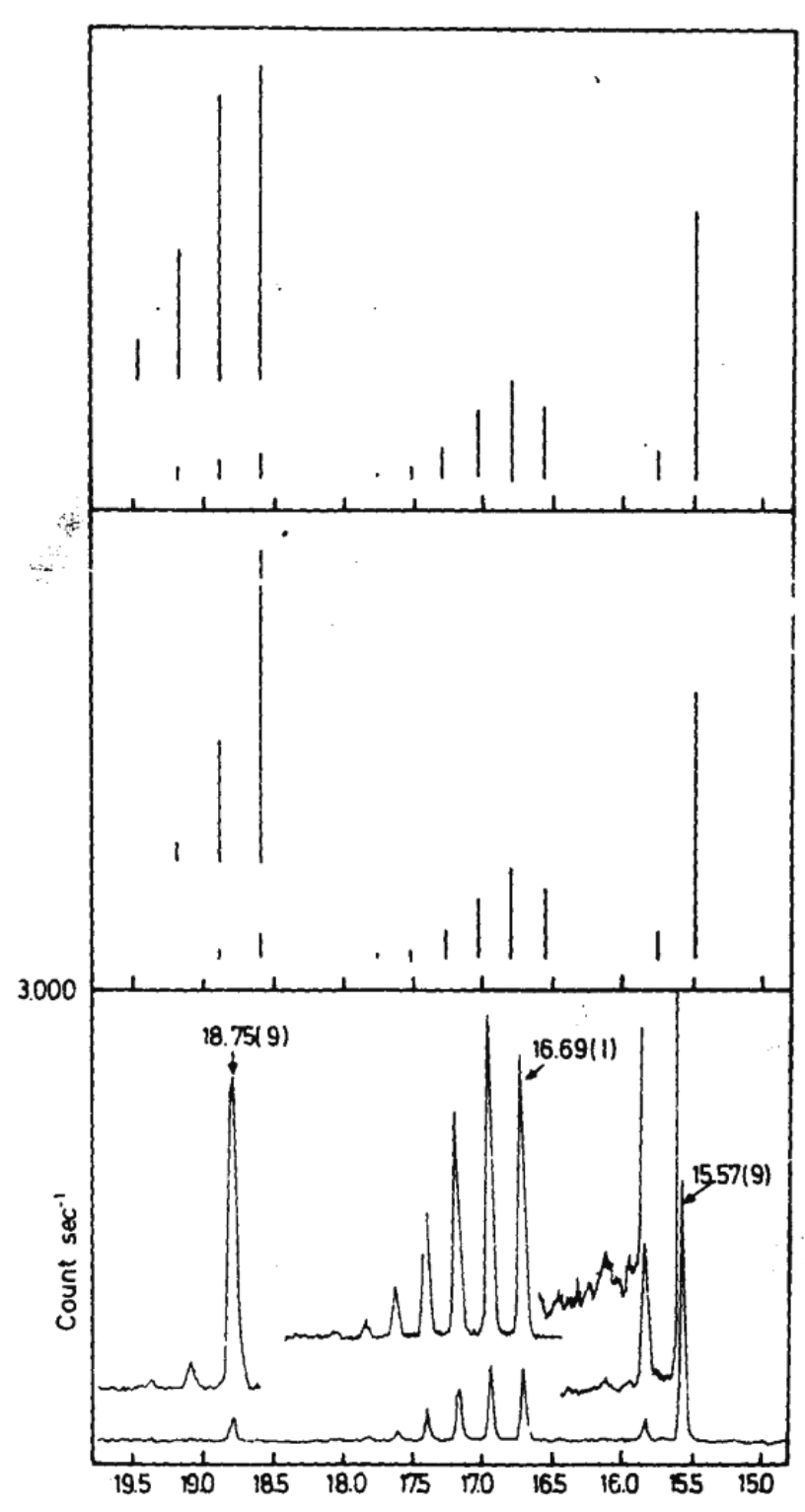

Fig. 3. Experimental and calculated PE spectra of $\mathrm{N}_{2}$. The uppermost spectrum is calculated with $\boldsymbol{S}^{0}$ and Eq. (54). The middle spectrum is calculated with values of S from (29) (see text and $[12,14])$.

In case the frequencies change considerably, the intensity distribution of the most intensive lines can analogously be well approximated by an effective parameter $\boldsymbol{S}$. In fig. 1 the experimental and calculated photoelectron spectra for the $\mathrm{N}_{2}$. molecule are presented. The uppermost spectrum is calculated with $\boldsymbol{S}^{0}$ (i.e. the constant $\mathrm{S}$ calculated with $\boldsymbol{g}^{0}$ ) and Eq. (54). The middle spectrum is calculated with values of S from Eq. (29). It is important to note that the original Green's functions and combined Green functions +DFT approach coincide in the scale of the figure. In a whole the agreement between the calculated spectrum (the corrected g ) and the experimental one is improved. Regarding the inclusion of the anharmonicites it should be mentioned that a theory can be generalized by means a standard normal coordinate expansion of the Hamiltonian to third and higher orders.

\section{CONCLUSIONS}

So, firstly we present a new combined theoretical approach to vibrational structure in molecular photoelectron spectra, which is based on the Green's function method and DFT approach. The density of states, which describe the vibrational structure in molecular photoelectron spectra, is calculated with the help of combined DFT-Green's-functions approach. It is important that the calculational procedure is significantly simplified with using the quasiparticle DFT formalism. In result, we believe that quite simple theory become a powerful tool in interpreting the vibrational structure of the molecular photoelectron spectra.

\section{References}

1. Turner D., Baker C., Baker A., Brunrile C., Molecular Photoelectron Spectroscopy, New York: Wiley. - 2005. - 540P.

2. Kaplan I.G., Theory of intermolecular interactions, World Sci.: Singapore, 2005. - 350P.

3. Wilson S., Handbook on Molecular Physics and Quantum Chemistry. - Chichester: Wiley. - 2007. - 700P.

4. Crawford T., Schaefer H.F., III, in Reviews of Computational Chemistry, eds. K. B. Lipkowitz and D.B. Boyd, New York: Wiley, 2000. - Vol.14. - P.33-136.

5. Nayak M., Chaudhuri R., Das.B., Ab initio calculation of electron-nucleus scalar-pseudo-scalar interaction constant $\mathrm{W}_{\mathrm{s}}$ in heavy polar molecules//Phys.Rev.A. - 2007. Vol.75. - P.022510.

6. Bartlett R.J., Musiał M.N., Coupled-cluster theory in quantum chemistry//Rev. Mod. Phys. - 2007. - Vol.79. - P.291328.

7. Grant I. P., Relativistic quantum theory of atoms and molecules. - N. - Y.:Springer, 2007. - 286P.

8. Abrikosov A., Gorkov L., Dzyaloshinskii E., Quantum Field Theoretical Methods in Statistical Physics, Oxford: Pergamon, 1995. - 400P.

9. Dorofeev D., Zon B.A., Kretinin I., Chernov V.E., Method of quantum defect Green's function for calculation of dynamic atomic polarizabilities// Optics and Spectr. - 2005. Vol. 99. - P.540-548.

10. Zheng R., Wei W., Shi Q., Density functional theory study on sum-frequency vibrational spectroscopy of arabinose chiral solutions// J. Phys. Chem. A. - 2009. - Vol.113. - P.157164.

11. Glushkov A.V., Relativistic quantum theory. Quantum mechanics of atomic systems. - Odessa: Astroprint, 2008. - P 800 .

12. Kцppel H., Domcke W., Cederbaum L.S., Green's function method in quantum chemistry// Adv. Chem. Phys. 1999. - Vol.57. - P.59-132.

13. Cederbaum L.S., Domcke W., On vibrational structure of photoelectron spectra by the Green's functions method// J.Chem. Phys. - 1994. - Vol.60. - P.2878-2896.

14. Glushkov A.V., New approach to theoretical definition of ionization potentials for molecules on the basis of Green's function method//Journ.of Phys.Chem. - 1999-Vol.66. P.2671-2677.

15. Kohn W., Sham L.J. Quantum density oscillations in an inhomogeneous electron gas//Phys. Rev. A. - 1999. Vol.137,N6. - P.1697-1706.

16. Gross E.G., Kohn W. Exchange-correlation functionals in density functional theory. - N-Y: Plenum, 2005. - 380P.;

17. The Fundamentals of Electron Density, Density Matrix and Density Functional Theory in Atoms, Molecules and the Solid State, Series: Progress in Theoretical Chemistry and Physics, Eds. Gidopoulos N.I. and Wilson S. - Amsterdam: Springer, 2004. - Vol.14, 244P.

18. Glushkov A.V., Relativistic and correlation effects in spectra of atomic systems.. - Odessa: Astroprint, 2006. - P 400.

19. Glushkov A.V., Khetselius O.Yu., Malinovskaya S.V., Optics and spectroscopy of cooperative laser-electron nuclear 
processes in atomic and molecular systems - new trend in quantum optics// Europ.Phys.Journ. ST. - 2008. Vol.160,N1. - P.195-204.

20. Glushkov A.V., Khetselius O.Yu., Malinovskaya S.V., Spectroscopy of cooperative laser-electron nuclear effects in multiatomic molecules// Molec. Physics . - 2008. - Vol.106. N9\&10. - P.1257-1260.

21. Glushkov A.V., Malinovskaya S.V., et al, Quantum stochastic modeling energy transfer and effect of rotational, V-T relaxation on multi-photon excitation and dissociation for molecules// Int. Journ. of Quantum Chemistry. - 2005. Vol.105-P.512-516.

22. Glushkov A.V., Malinovskaya S.V., Lovett L. et al, Green's function method in quantum chemistry: New algorithm for the Dirac equation with complex energy and Fermi-model nuclear potential// Int. Journ. of Quantum Chemistry. 2009. - Vol.109. - N10. - P.1331-1345.
23. Glushkov A.V., Florko T.A., Lovett L et al, Gauge-invariant QED perturbation theory approach to nuclear electric quadrupole moments, hyperfine structure constants for heavy atoms and ions// Frontiers in Quantum Systems in Chemistry and Physics . - 2008. - Vol.18. - P.505-522.

24. Appel H., Gross E.K.U., Time-dependent natural orbitals and occupation numbers//Phys. Rev. Lett. - 2009. - Vol.102. P.024331.

25. Glushkov A.V., Lepikh Ya.I., Fedchuk A.P., Loboda A.V., Lovett L., Electrodynamical and quantum - chemical approaches to modelling the electrochemical and catalytic processes on metals, metal alloys and semiconductors//Int. Journ. of Quantum Chemistry. - 2009. - Vol.111, N4, to be printed.

26. Ivanova E.P., Ivanov L.N., Glushkov A.V., Kramida A.E., High-order corrections in the relativistic perturbation theory with model zeroth approximation//Physica Scripta. 1999. - V.32. - P.512-524

UDC 539.19+539.182

A. P. Fedchuk, A. V. Glushkov, Ya. I. Lepikh, A. V. Loboda

THE GREEN'S FUNCTIONS AND DENSITY FUNCTIONAL APPROACH TO VIBRATIONAL STRUCTURE IN THE PHOTOELECTRON SPECTRA OF MOLECULES

Abstract

We present the basis's of the new combined theoretical approach to vibrational structure in photoelectron spectra of molecules. The approach is based on the Green's function method and density functional theory (DFT). The density of states, which describe the vibrational structure in photoelectron spectra, is defined with the use of combined DFT-Green's-functions approach and is well approximated by using only first order coupling constants in the one-particle approximation. It is important that the calculational procedure is significantly simplified with using the DFT.

Key words: photoelectron spectra, Green's functions method, density functional theory.

УДК 539.19+539.182

А. В. Глушков, Я. И. Лепих, А. П. Федчук, А. В. Лобода

\section{МЕТОД ФУНКЦИЙ ГРИНА И ФУНКЦИОНАЛА ПЛОТНОСТИ В ОПРЕДЕЛЕНИИ КОЛЕБАТЕЛЬНОЙ СТРУКТУРЫ ФОТОЭЛЕКТРОННЫХ СПЕКТРОВ МОЛЕКУЛ}

\section{Резюме}

Изложены основы нового комбинированного теоретического метода описания колебательной структуры для фотоэлектронных спектров молекул, который базируется на методе функций Грина и теории функционала плотности (ТФП). Плотность состояний, описывающая колебательную структуру спектра, определяется с использованием метода функций Грина и квазичастичной ТФП и приемлемо аппроксимируется с использованием констант связи только первого порядка уже в одночастичном приближении.

Ключевые слова: фотоэлектронный спектр, метод функций Грина, теория функционала плотности.

\section{УДК 539.19+539.182}

О. В. Глушков, Я. І. Лепіх, О. П. Федчук, А. В. Лобода

\section{МЕТОД ФУНКЦІЙ ГРІНА І ФУНКЦІОНАЛУ ГУСТИНИ У ВИЗНАЧЕННІ ВІБРАЦІЙНОЇ СТРУКТУРИ ФОТОЕЛЕКТРОННИХ СПЕКТРІВ МОЛЕКУЛ}

\section{Резюме}

Викладені основи нового комбінованого теоретичного методу опису вібраційної структури для фотоелектронних спектрів молекул, який базується на методі функцій Гріна і теорії функціоналу густини (ТФГ). Густина станів, що описує вібраційну структуру спектра, визначається з використанням методу функцій Гріна та квазічастинкової ТФГ і прийнятно апроксимується з використанням сталих зв’язку тільки першого порядку навіть у одночастинковому наближенні.

Ключові слова: фотоелектронний спектр, метод функцій Гріна, теорія функціонала густини. 